\title{
Comportamiento Dispersivo de la Energía Elástica en Medios Granulares
}

\author{
Juan D. Morinson (1), Franklin E. Peniche(2), José A. Molina(3) \\ Universidad de Córdoba, Facultad de Ciencias Básicas, Carrera 6 No. 76-103, km 3 vía Cereté - Córdoba, Colombia \\ (1) Departamento de Física, Grupo de investigación GEMASGRA (e-mail: jdmorinson1984@hotmail.com); \\ (2) Departamento de Física, Grupo de investigación GAMASCO (e-mail: fpenichedavf@gmail.com); \\ (3) Departamento de Física (e-mail: profesorjosemolina@hotmail.es)
}

Recibido Oct. 13, 2014; Aceptado Dic. 23, 2014; Versión final recibida Mar. 1, 2015

\begin{abstract}
Resumen
Este trabajo muestra el comportamiento de la energía de modos normales de vibración, que constituyen una onda elástica, cuando esta experimenta dispersión en un medio granular. Para esto, se grafican patrones de radiación dispersada, entre diversos pares de modos, los que son analizados destacando las principales direcciones de dispersión. Para lo anterior, se utilizó la sección eficaz de dispersión modal W(iki,jkj) obtenida por Trujillo y colaboradores en 2013. Los modos normales utilizados, son las soluciones del problema de vibraciones libres de un plato elástico, homogéneo e isotrópico, es decir, modos de cizalla horizontal y Lamb, los cuales a su vez pueden ser simétricos o antisimétricos. Este trabajo contribuye al entendimiento del comportamiento de la estructura interna de los medios granulares, como también a potenciales aplicaciones en campos tales como la mecánica de suelos, geofísica, sismología y exploración petrolera, entre otras.
\end{abstract}

Palabras clave: energía elástica, sección eficaz, modos normales, onda elástica, medio granular

\section{Dispersive Behavior of Elastic Energy in Granular Media}

\begin{abstract}
This paper shows the behavior of the energy of normal modes of vibration, which constitute an elastic wave, when it undergoes dispersion in a granular media. For this, scattered radiation patterns between different pairs of modes are plotted and analyzed, highlighting the main directions of dispersion. For this, cross sections of modal dispersion W(iki, jkj) obtained by Trujillo and coworkers in 2013 was used. The normal modes implemented are the solutions of the problem of free vibrations of an elastic, homogeneous and isotropic plate, namely, horizontal shear modes and Lamb, which in turn can be symmetric or antisymmetric. This work aims to contribute to the understanding of the behavior of the internal structure of granular media, as well as potential applications in fields such as, soils mechanics, geophysics, seismology, and oil exploration, among others.
\end{abstract}

Keywords: elastic energy, cross section, normal modes, elastic wave, granular media 


\section{INTRODUCCIÓN}

En las últimas décadas, los sistemas granulares han despertado en la comunidad científica gran interés y dedicación, debido a su llamativo comportamiento, ancho espectro de acción, gran interdisciplinariedad conceptual y su gran aplicabilidad para el ser humano. Se considera como sistema granular, a todo conjunto de partículas sólidas macroscópicas discretas, que interactúan mediante fuerzas de contacto repulsivo y de corto alcance (Trujillo et al., 2013). El tamaño de estas partículas va desde el orden de los micrones, en dónde la agitación térmica es despreciable, hasta el orden de los metros. En la vida cotidiana, existen muchos ejemplos clásicos de estos sistemas, entre esos, la arena, la gravilla, los granos de arroz, la azúcar, entre otros (Forterre y Pouliquen, 2011).

Los sistemas granulares pueden comportarse de diferentes formas: Como estado sólido, es decir, pueden ser lo suficientemente fuertes como para sostener edificaciones, también pueden comportarse como fluidos, como por ejemplo, en un reloj de arena, o pueden transportarse por el viento, para crear dunas en el desierto. Esta variedad de comportamientos, representa una de las dificultades de la física de medios granulares. Las investigaciones en este campo de la física, se han ido incrementando, debido a la diversidad de aplicaciones en procesos industriales y especialmente en Geofísica, para la predicción y descripción de desastres naturales, como los deslizamientos de tierra, formación de avalanchas, entre otros (Forterre y Pouliquen, 2011). El modelamiento de estos sistemas de muchas partículas, es bastante complicado, debido a que los granos tienen tamaños, densidades y formas diferentes, lo cual conlleva a comportamientos mecánicos complejos. Así, el planteamiento de modelos teóricos para la descripción y entendimiento de estos sistemas, es un trabajo desafiante y bastante arduo.

En este trabajo, se pretende mostrar cómo se comporta de manera dispersiva la energía elástica en un medio granular, en donde se asume que la onda elástica total, se puede expresar como la superposición de los modos normales que surgen de la solución del problema de vibraciones libres de un plato elástico homogéneo e isotrópico. Para la obtención de tal logro, se tendrán en cuenta los resultados teóricos encontrados recientemente por Trujillo y colaboradores en la referencia (Trujillo et al., 2011), en donde se propone un modelo teórico basado en primeros principios, que pretende describir el comportamiento de la propagación de ondas elásticas a través de medios granulares. En este modelo, ellos asumen el medio granular como un medio continuo desordenado, el cual, se entiende como un plato elástico homogéneo e isotrópico en el que se introduce posteriormente desorden a través de pequeñas fluctuaciones en cada punto, ya sea en la densidad local o en las constantes elásticas a través de los coeficientes de Lamé locales. Proponen una expresión analítica para la sección eficaz de conversión modal $\mathrm{W}\left(\mathrm{ik}_{\mathrm{j}}, \mathrm{j} \mathrm{k}_{\mathrm{j}}\right)$ válida en este medio granular, la cual da cuenta de cómo la energía del modo normal de vibración i-ésimo con vector de onda $k_{i}$, es dispersada convirtiéndose en energía del modo de vibración j-ésimo con vector de onda $k_{j}$, cuando la onda elástica experimenta una dispersión dentro del medio granular debido a su desorden intrínseco. Los subíndices i y j representan distintas ramas de dispersión que determinan todos los posibles modos normales de vibración del plato elástico, homogéneo e isotrópico, los cuales son dados en la referencias (Weaver, 1984; Moreau et al., 2011). Dichos modos son de dos clases: modos de cizalla horizontal (SH) y modos Lamb, estos últimos son una combinación de deformaciones de cizalla y de compresión. Todos estos modos a su vez pueden ser simétricos o antisimétricos, dependiendo de la forma en que vibra el plato con respecto al plano medio de la misma.

Además, se tendrán en cuenta para la realización de los cálculos, parámetros similares a los usados en los experimentos realizados por Jia y colaboradores, dados en las referencias (Jia et al., 2009; Jia, 2004), en dónde ellos demuestran que el ultrasonido ofrece una valiosa técnica para investigar las propiedades mecánicas de un medio granular. Como uno de los resultados principales de los mencionados experimentos, ellos encuentran que la intensidad de las ondas de ultrasonido transmitidas en función del tiempo I(t), a través del paquete granular, satisface una ecuación de difusión, concluyendo de esta manera que dichas ondas se propagan dispersándose múltiplemente en una forma difusiva normal. La razón de usar estos parámetros es poder realizar comparaciones de los resultados aquí presentados y otros que de aquí se deriven con los mencionados experimentos en trabajos futuros de esta línea.

De acuerdo a lo mencionado anteriormente, en el presente trabajo se mostrará el cálculo de casos particulares de $\mathrm{W}\left(\mathrm{ik}_{\mathrm{i}}, \mathrm{j} \mathrm{k}_{\mathrm{j}}\right)$ y sus correspondientes gráficas polares, para conversión de modos de vibración $\mathrm{SH}$ y Lamb, simétricos y antisimétricos, respectivamente, en las cuales se analizará cómo es el comportamiento en cada dispersión dentro del medio granular. Con este trabajo se pretende contribuir al entendimiento de la respuesta mecánica de medios granulares a perturbaciones externas, mediante una mejor comprensión de cómo se propaga la energía elástica en estos medios, lo cual no solo tiene interés, desde el punto de vista físico, sino también en numerosas actividades científicas, tales como la mecánica de suelos y la geofísica, además de su importancia para la industria (por ejemplo en la exploración petrolera, en la construcción para la estabilidad estructural, etcétera). 


\section{MATERIALES Y MÉTODOS}

\section{Sección eficaz de dispersión modal}

Como se menciono en la introducción, para realizar los cálculos de secciones eficaces de dispersión modal, se tendrá en cuenta la ecuación propuesta en el modelo de Trujillo y colaboradores (Trujillo et al., 2011), es decir,

$W\left(i k_{i}, j k_{j}\right)=\int_{0}^{h} d z \sigma^{2}\left|\frac{9 \lambda_{0}}{32 \delta_{0}}\left(\nabla \cdot u_{i k_{i}}\right)^{*}\left(\nabla \cdot u_{j k_{j}}\right)+\frac{\mu_{0}}{2 \delta_{0}} S_{i k_{i}}^{*}: S_{j k_{j}}\right|^{2}+\int_{0}^{h} d z \sigma^{2}\left|\frac{\mu_{0}}{4 \delta_{0}^{2}} S_{j k_{j}}^{*}: S_{j k_{j}}\left(\nabla \cdot u_{i k_{j}}\right)^{\star}\right|^{2}$

Donde $\mathrm{h}$ es el espesor del plato granular, $\mathrm{z}$ representa la coordenada espacial a lo largo del espesor de la placa, $u_{i k_{i}}$ y $u_{j k_{j}}$ son los modos normales de vibración del plato homogéneo e isotrópico, $\sigma$ es la intensidad del desorden, el cual se considera como $\delta$-correlacionado, $\lambda_{0}$ y $\mu_{0}$, son los coeficientes de Lamé promedio o asociados al plato homogéneo, $\delta_{\mathrm{o}}$ es la compresión de volumen promedio del sistema, y $S_{\mathrm{i} k_{i}}^{*}: S_{\mathrm{j} k_{j}}$, es el producto doble punto definido por:

$S_{\mathrm{i} k_{i}}^{*}: S_{\mathrm{j} k_{j}}=\sum_{n} \sum_{m} \mathrm{u}_{\mathrm{mn}_{\mathrm{ik}}}^{*} \mathrm{u}_{\mathrm{nm}} k_{\mathrm{j}}$

En este caso, $u_{m n}^{*}{ }_{i k_{i}} y u_{n_{j} k_{j}}$, son los tensores de deformación asociados a los modos normales de vibración $i k_{i}$ y $j k_{j}$. Se debe recordar que el tensor de deformación se calcula de la expresión, $u_{n m}=u_{m n}=1 / 2\left(\partial_{m} u_{n}+\partial_{n} u_{m}\right)$.

El método de modos normales de vibración es ampliamente utilizado en diversos campos como por ejemplo: en físicoquímica molecular (Mills y Robiette., 1985; Lawton y Child., 1979; Carter et al., 1998; Hutchinson et al., 1983), en el estudio de la dinámica conformacional de proteínas (Go et al., 1983), en el desarrollo de dispositivos de procesamiento de señales acústicas (Li y Melngailis., 1975), en astrofísica (Harrison, 1967), etcétera.

Dependiendo del sistema de vibración estudiado y del tipo de elasticidad considerada, esto es elasticidad lineal o no-lineal, existen diferentes métodos o estrategias para determinar la forma de los modos normales de vibración (S. Shaw and C. Pierre, 1991; Weaver, 1984; Moreau et al., 2011). En este trabajo por simplicidad se consideran los modos normales de vibración libre de un plato homogéneo e isotrópico que satisface el modelo de elasticidad lineal y que están dados en las referencias (Weaver, 1984; Moreau et al., 2011). Se deja para futuros trabajos en esta línea la obtención de los modos normales del plato bajo el modelo de elasticidad no-lineal de Jiang y Liu (Jiang y Liu., 2007) el cual describe mejor el comportamiento granular. La forma matemática de los modos aquí considerados se presentan a continuación (Weaver, 1984; Moreau et al., 2011).

\section{Modos SH}

$u=N_{b} \nabla \times\left(\hat{z} f_{b}(r)\right) V_{b}(z) e^{-i \omega t}$

Donde $N_{b}=1 / \sqrt{A}$ es la constante de normalización; $A$ es el área del plato,ż es el vector unitario asociado a la coordenada espacial $z, y \omega$ es la frecuencia central del pulso ultrasónico entrante al paquete granular y. En este caso, b denota las ramas de dispersión i o j, y $\mathrm{f}_{\mathrm{b}}(\mathrm{r})$ tiene la forma:

$f_{b}(r)=e^{i k_{b} \cdot r}$

En este caso, $i=\sqrt{-1}$ y $k_{b}$.r es el producto punto entre el vector de onda $k_{b}$ y el vector posición $r$. Para el caso de modos normales de vibración $\mathrm{SH}$ simétricos, la función $\mathrm{V}_{\mathrm{b}}(\mathrm{z})$ tiene la forma,

$V_{b}(z)=\cos n \pi z / h$

y para los modos $\mathrm{SH}$ antisimétricos,

$V_{b}(z)=\sin (n+1 / 2) \pi z / h$

En las ecuaciones (5) y (6),n especifica el número de la rama de dispersión, el cual está implícito en b, y toma valores enteros de $n=0,1,2,3, \ldots$. 
Las relaciones de dispersión para las ramas simétricas y antisimétricas, tienen la forma,

$\mathrm{k}_{\mathrm{b}}^{2}=\frac{\omega^{2}}{\mathrm{c}_{\mathrm{s}}^{2}}-\left(\frac{\mathrm{n} \pi}{\mathrm{h}}\right)^{2}$

$\mathrm{k}_{\mathrm{b}}^{2}=\frac{\omega^{2}}{\mathrm{Cs}_{\mathrm{s}}^{2}}-\left(\frac{(\mathrm{n}+1 / 2) \pi}{\mathrm{h}}\right)^{2}$

En este caso, $\mathrm{c}_{\mathrm{s}}$ es la rapidez con que se propaga la onda de cizalla.

Modos Lamb

$u=N_{b}\left[\hat{z} W_{b}(z) f_{b}(r)+U_{b}(z) \nabla f_{b}(r)\right]$

Para el caso de modos Lamb antisimétrico,

$U_{b}(z)=\left[2 k_{b}{ }^{2} \beta_{b} \sin \beta_{b} h\right] \sin \alpha_{b} z-\left[\beta_{b}\left(k_{b}{ }^{2}-\beta_{b}{ }^{2}\right) \sin \alpha_{b} h\right] \sin \beta_{b} z$

$W_{b}(z)=\left[2 \alpha_{b} k_{b}{ }^{2} \beta_{b} \sin \beta_{b} h\right] \cos \alpha_{b} z+\left[k_{b}{ }^{2}\left(k_{b}{ }^{2}-\beta_{b}{ }^{2}\right) \sin \alpha_{b} h\right] \cos \beta_{b} z$

Los números de onda vertical, tienen la forma,

$\alpha_{b}^{2}=\frac{\omega^{2}}{c_{d}^{2}}-k_{b}^{2}, \quad \beta_{b}^{2}=\frac{\omega^{2}}{c_{s}^{2}}-k_{b}^{2}$

En este caso, $c_{d}$ representa a la rapidez con que se propaga la onda de compresión en el plato. Las relaciones de dispersión para ondas Lamb antisimétrica tienen la forma,

$\left(k_{b}{ }^{2}-\beta_{b}{ }^{2}\right)^{2} \cos \beta_{b} h \sin \alpha_{b} h+4 a_{b} \beta_{b} k_{b}^{2} \sin \beta_{b} h \cos \alpha_{b} h=0$

Es fácil observar que la ecuación (13) es de tipo trascendental, es decir, para encontrar las soluciones de los números de onda $\mathrm{k}_{\mathrm{b}}$, cuando se fija el valor numérico de la frecuencia central $\omega$, se debe hacer uso de métodos numéricos a través de la máquina computacional. Para el caso Lamb simétrico se intercambia las funciones sin y cos en las ecuaciones (10), (11) y (13), y se intercambia $U$ con $-U$ en la ecuación (10).

\section{RESULTADOS Y DISCUSIÓN}

Para realizar los cálculos de $\mathrm{W}\left(i k_{i}, j k_{j}\right)$, inicialmente, se definen los valores numéricos para las constantes de elasticidad y parámetros que aparecen en la ecuación (1), estos son tomados del experimento de Jia, $X$. (Jia, 2004), esto es, $c_{s}=450 \mathrm{~m} / \mathrm{s}, c_{d}=10^{3} \mathrm{~m} / \mathrm{s}, \mathrm{h}=11.4 \times 10^{-3} \mathrm{~m}, \omega=10^{6} \mathrm{mHz}$, de donde se deduce que, $\lambda_{0}=9.37 \times 10^{8} \mathrm{~Pa}, \delta_{0}=6.52 \times 10^{-4}, \mu_{0}=3.19 \times 10^{8} \mathrm{~Pa}$. Seguidamente, se reemplaza estos valores numéricos en las relaciones de dispersión para modos de vibración SH y Lamb, dadas en las ecuaciones (7), (8) y (13), con el fin de obtener el número de formas en que vibra el plato granular a una frecuencia central $\omega_{\mathrm{r}}$. Para este caso particular, se adimensionalizaron las relaciones de dispersión dadas por las ecuaciones [(7), (8) y (13)], haciendo uso de variables reducidas propuestas en la referencia (Weaver, 1984), esto es, $k_{b}=\left(\frac{\pi}{h}\right) k_{r}$ y $\omega_{b}=\left(\frac{\pi c_{s}}{h}\right) \omega_{r} ; k_{r}$ y $\omega_{r}$, son las nuevas variables reducidas. De esta forma, se obtiene que el número de modos normales de vibración del plato son cuarenta (40), es decir, el número de secciones eficaces de dispersión modal $\mathrm{W}\left(\mathrm{i} k_{i}, \mathrm{j} \mathrm{k}_{\mathrm{j}}\right)$ que se deben calcular son mil seiscientos (1600), lo cual es un tratamiento muy dispendioso y extenso, por lo que aquí se mostrará solamente el cálculo de algunos casos particulares.

Luego, se ordenan los modos normales de vibración del plato granular, para saber quién es el modo $1,2,3, \ldots, 40$, esto se logra ordenando en forma descendente los valores numéricos obtenidos para los números de onda $\mathrm{k}_{\mathrm{i}}$. Así, el orden modal del plato granular es:\{shs, sha, shs, sha, shs, sha, shs, sha, shs, sha, shs, sha, shs, sha, shs, sha, shs, sha, shs, sha, shs, sha, shs, lambs, lambas, lambs, lambas, sha, lambas, lambs, lambas, lambs, lambas, lambs, shs, lambs, lambas, sha, lambas, lambs\}, en este caso, se ha utilizado las abreviaturas shs para representar a los modos normales de vibración SH simétrico, shaa los $\mathrm{SH}$ antisimétrico, lambs para los Lamb simétrico y lambas para los Lamb antisimétrico. En este trabajo, solo 
se mostrará algunos casos particulares para $W\left(i_{i}, j k_{j}\right)$, esto es, $W_{11}, W_{22}, W_{12}, W_{38}, W_{124}, W_{1331}, W_{826}$, $\mathrm{W}_{437}, \mathrm{~W}_{2424}, \mathrm{~W}_{2411}, \mathrm{~W}_{246}, \mathrm{~W}_{2427} \cdot \mathrm{W}_{2515}, \mathrm{~W}_{254}, \mathrm{~W}_{2524}$ y $\mathrm{W}_{2527}$.

En este caso, el elemento $\mathrm{W}_{11}$, corresponde al primer modo $\mathrm{SH}$ simétrico con el primer modo $\mathrm{SH}$ simétrico. Para calcularlo se reemplaza la ecuación (3) en (1), como también el valor de $k_{1}$, es decir, el primer número de onda $\mathrm{k}$, y luego normalizando se obtiene,

$\mathrm{W}_{11} / \Sigma_{11}=5.36 \times 10^{-1}+\left(5.36 \times 10^{-1}\right) \cos [4 \theta]$

Donde $\Sigma_{11}$ es la sección eficaz total de dispersión para el primer modo SH simétrico con el primer modo SH simétrico, la cual se puede calcular, $\Sigma_{11}=\int_{-1}^{1} W_{11}(X) d x ; \quad X=\cos \theta$ y $\theta$ es el ángulo de dispersión medido con respecto a la dirección de incidencia del pulso ultrasónico.

Los otros casos particulares para las secciones eficaces de dispersión, se obtienen siguiendo un camino similar, esto es,

SH Antisimétrico - SH Antisimétrico

$\mathrm{W}_{22} / \Sigma_{22}=\left(2.62 \times 10^{-6}\right) \cos [\theta]^{2}+\left(1.12 \times 10^{-3}\right) \cos [\theta] \cos [2 \theta]+(1.07) \cos [2 \theta]^{2}$

SH Simétrico - SH Antisimétrico

$\mathrm{W}_{12} / \Sigma_{12}=\mathrm{W}_{21} / \Sigma_{21}=5.36 \times 10^{-1}+\left(5.36 \times 10^{-1}\right) \cos [4 \theta]$

SH simétrico - Lamb Simétrico

$\mathrm{W}_{124} / \Sigma_{124}=\left(9.38 \times 10^{-1}\right) \sin [2 \theta]^{2}$

SH Simétrico - Lamb Antisimétrico

$\mathrm{W}_{1331} / \Sigma_{1331}=\left(1.3+\left(5.28 \times 10^{-2}\right) \cos [\theta]+\left(9.24 \times 10^{-1}\right) \cos [2 \theta]\right) \sin [\theta]^{2}$

SH Antisimétrico - Lamb Simétrico

$\mathrm{W}_{826} / \Sigma_{826}=\left(1.77-\left(4.75 \times 10^{-3}\right) \cos [\theta]+1.7 \cos [2 \theta]\right) \sin [\theta]^{2}$

SH Antisimétrico - Lamb Antisimétrico

$\mathrm{W}_{437} / \Sigma_{437}=\left(-5.9 \times 10^{-4}\right)\left(-2.75 \times 10^{3}+\cos [\theta]-\left(2.47 \times 10^{3}\right) \cos [2 \theta]\right) \sin [\theta]^{2}$

Lamb Simétrico - Lamb Simétrico

$$
\begin{aligned}
& \mathrm{W}_{2424} / \Sigma_{2424}=5 \times 10^{-1}+\left(3.34 \times 10^{-37}\right) \cos [\theta]+\left(2.39 \times 10^{-36}\right) \cos [2 \theta]+\left(3.46 \times 10^{-38}\right) \cos [3 \theta] \\
& +\left(1.58 \times 10^{-37}\right) \cos [4 \theta]
\end{aligned}
$$

Lamb Simétrico - SH Simétrico

$W_{2411} / \Sigma_{2411}=2.5 \times 10^{-1}+\left(3.39 \times 10^{-16}-\left(1.44 \times 10^{-17}\right) \cos [\theta]+\left(3.32 \times 10^{-16}\right) \cos [2 \theta]\right) \sin [\theta]^{2}$

Lamb Simétrico - SH Antisimétrico

$$
\mathrm{W}_{246} / \Sigma_{246}=5 \times 10^{-1}+\left(6.33 \times 10^{-16}+\left(1.9 \times 10^{-18}\right) \cos [\theta]+\left(6.31 \times 10^{-16}\right) \cos [2 \theta]\right) \sin [\theta]^{2}
$$

Lamb Simétrico - Lamb Antisimétrico

$\mathrm{W}_{24} 27 / \Sigma_{2427}=5 \times 10^{-1}-\left(8.9 \times 10^{-45}\right) \cos [\theta]+\left(9.96 \times 10^{-44}\right) \cos [2 \theta]-\left(1.53 \times 10^{-45}\right) \cos [3 \theta]$ 
$+\left(1.06 \times 10^{-44}\right) \cos [4 \theta]$

Lamb Antisimétrico - SH Simétrico

$W_{2515} / \Sigma_{2515}=1.26 \times 10^{43}-\left(2.24 \times 10^{-1}\right) \cos [\theta]+2.51 \cos [2 \theta]-\left(3.86 \times 10^{-2}\right) \cos [3 \theta]+\left(2.67 \times 10^{-1}\right) \cos [4 \theta]$

Lamb Antisimétrico - SH Antisimétrico

$$
W_{254} / \Sigma_{254}=5 \times 10^{-1}+\left(1.06 \times 10^{-21}+\left(4.2 \times 10^{-24}\right) \cos [\theta]+\left(1.06 \times 10^{-21}\right) \cos [2 \theta]\right) \sin [\theta]^{2}
$$

Lamb Antisimétrico - Lamb Simétrico

$$
\mathrm{W}_{2524} / \Sigma_{2524}=5 \times 10^{-1}-\left(7.68 \times 10^{-44}\right) \cos [\theta]+\left(3.86 \times 10^{-42}\right) \cos [2 \theta]-\left(3.09 \times 10^{-45}\right) \cos [3 \theta]+\left(2.52 \times 10^{-43}\right) \cos [4 \theta]
$$

Lamb Antisimétrico - Lamb Antisimétrico

$W_{2527} / \Sigma_{2527}=5 \times 10^{-1}+\left(2.69 \times 10^{-44}\right) \cos [\theta]+\left(1.32 \times 10^{-43}\right) \cos [2 \theta]+\left(3.11 \times 10^{-45}\right) \cos [3 \theta]+\left(1.29 \times 10^{-44}\right) \cos [4 \theta]$

Graficas polares de las secciones eficaces diferenciales de dispersión modal

Las gráficas polares de las secciones eficaces de dispersión normalizadas, dan cuenta de las direcciones que tomará la energía elástica, cuando sufre cada dispersión dentro del sistema .Para graficar los radios polares dados en las ecuaciones [(14) - (28)], se asume que el dispersor está en el origen de coordenadas, y que la onda incide desde la izquierda en un ángulo $\theta_{\mathrm{i}}=0^{\circ}$, con respecto a la horizontal. En la Figura 1 , se puede observar que la cantidad de energía del primer modo SH simétrico incidente que se dispersa en energía del primer modo SH simétrico $\left(\mathrm{W}_{11} / \Sigma_{11}\right)$, es la misma hacia adelante y hacia atrás, que para ángulos rectos con respecto a la dirección de incidencia, pero también se puede observar que para $45^{\circ}$ no hay dispersión. Un comportamiento similar se presenta para los casos de conversión del primer modo $\mathrm{SH}$ antisimétrico - primer modo SH antisimétrico $\left(\mathrm{W}_{22} / \Sigma_{22}\right)$ y también para el primer modo SH simétrico - primer modo $\mathrm{SH}$ antisimétrico $\left(\mathrm{W}_{12} / \Sigma_{12}=\mathrm{W}_{21} / \Sigma_{21}\right)$, respectivamente. Esto se justifica debido a la simetría que existe en la ecuación (1) para conversión de modos SH simétrico - SH antisimétrico o viceversa.

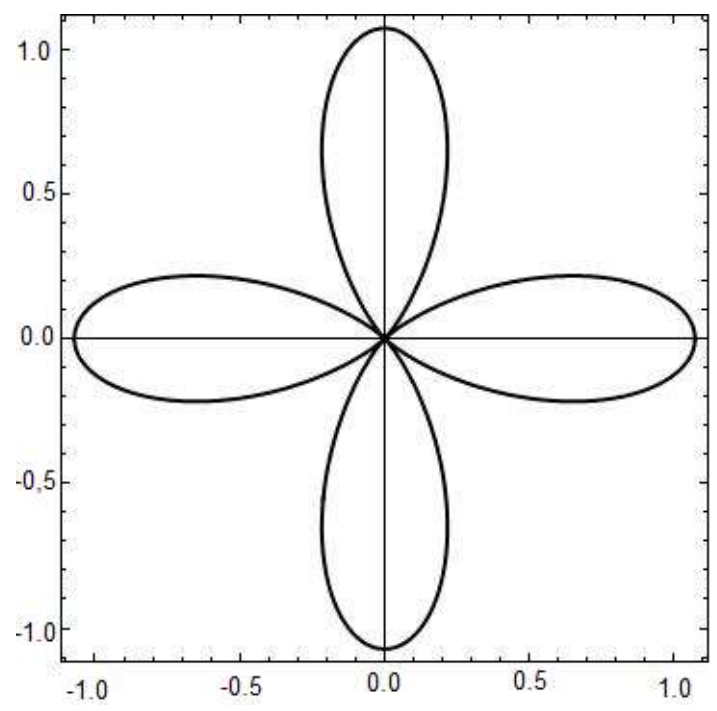

Fig. 1: Gráfica polar de las secciones eficaz de dispersión modal normalizadas $W_{11} / \Sigma_{11}, W_{22} / \Sigma_{22}$, $\mathrm{W}_{12} / \Sigma_{12}, \mathrm{~W}_{21} / \Sigma_{21}$, cuando $\theta \in[0,2 \pi]$, dada por las ecuaciones [(14) - (16)].

En la Figura 2 se muestra el patrón de radiación de energía elástica para conversión del primer modo SH simétrico - al primer modo Lamb simétrico $\left(\mathrm{W}_{124} / \Sigma_{124}\right)$. Se puede observar que la amplitud de dispersión hacia adelante y hacia atrás, como también para ángulos rectos con respecto a la dirección de incidencia es nula, y que para ángulos de $45^{\circ}, 135^{\circ}, 225^{\circ}$ y $315^{\circ}$ con respecto a la dirección de incidencia, la amplitud del radio polar de dispersión es máximo. 


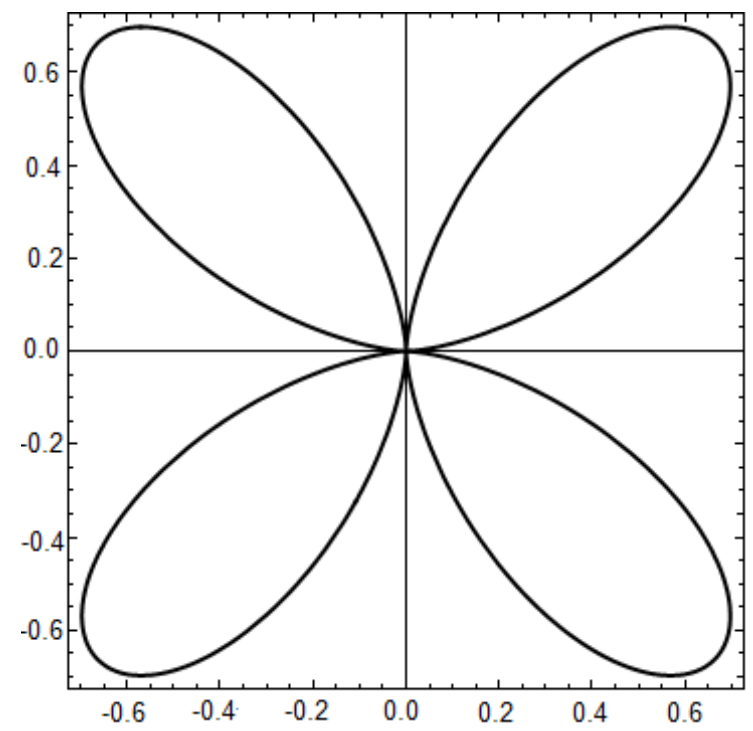

Fig. 2: Muestra la gráfica polar de la sección eficaz $W_{124} / \Sigma_{124}$ normalizada para el primer modo SH simétrico con el primer modo Lamb simétrico dada por la ecuación (17) cuando $\theta \in[0,2 \pi]$.

En la Figura 3 se observa que la amplitud del patrón de radiación para conversión del séptimo modo SH

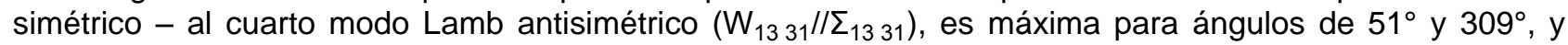
también para ángulos de $129^{\circ}$ y $231^{\circ}$, con respecto a la dirección de incidencia. Es claro que hacia adelante y hacia atrás la dispersión es nula.

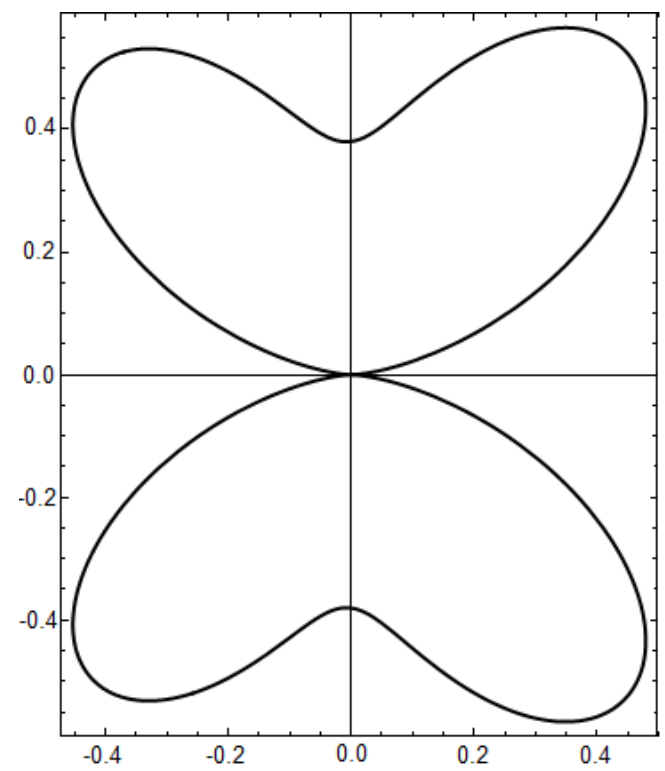

Fig. 3: Gráfica polar de la sección eficaz de dispersión modal normalizada $W_{1331} / / \Sigma_{1331}$ para el séptimo modo SH simétrico con el cuarto modo Lamb antisimétrico cuando $\theta \in[0,2 \pi]$, dada por la ecuación (18).

La Figura 4 muestra el radio polar de conversión del cuarto modo SH antisimétrico - al segundo modo Lamb simétrico $\left(\mathrm{W}_{826} / \Sigma_{826}\right)$, se puede observar que la dispersión de energía es máxima para ángulos de $46^{\circ}$ y $314^{\circ}$, y para ángulos de $134^{\circ}$ y $226^{\circ}$, con respecto a la dirección de incidencia, como también es fácil observar que no hay dispersión para $0^{\circ}$ y $180^{\circ}$. El radio polar de conversión modal, para el segundo modo $\mathrm{SH}$ antisimétrico - al sexto modo Lamb antisimétrico $\left(\mathrm{W}_{437} / \Sigma_{437}\right)$, se muestra en la Figura 5 . Se puede observar máximo de amplitud de dispersión para ángulos de $47^{\circ}$ y $313^{\circ}$, como también para $133^{\circ}$ y $227^{\circ}$, con respecto a la dirección de incidencia. Para ángulos de $0^{\circ}$ y $180^{\circ}$, la dispersión es nula. 


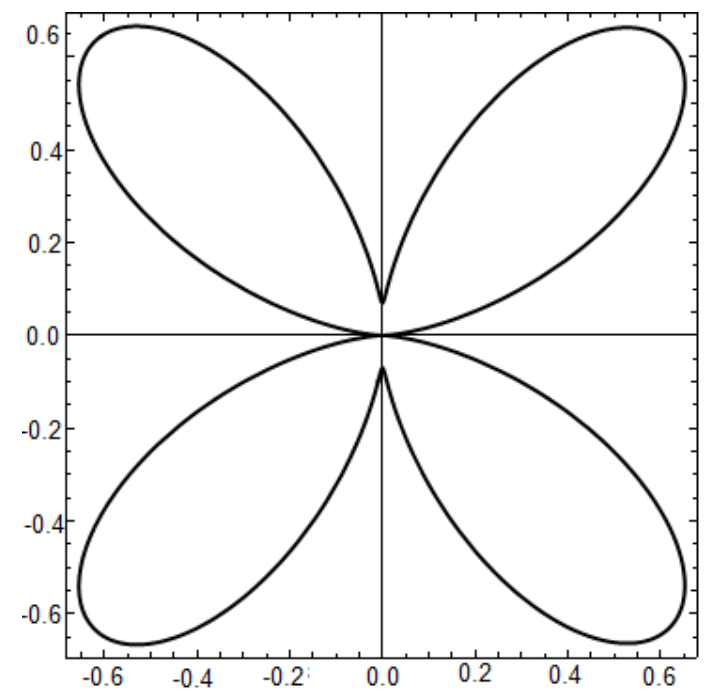

Fig. 4: Gráfica polar de la sección eficaz de dispersión modal normalizada $W_{826} / \Sigma_{826}$ para el cuarto modo $\mathrm{SH}$ antisimétrico con el segundo modo Lamb simétrico cuando $\theta \in[0,2 \pi]$, dada por la ecuación (19).

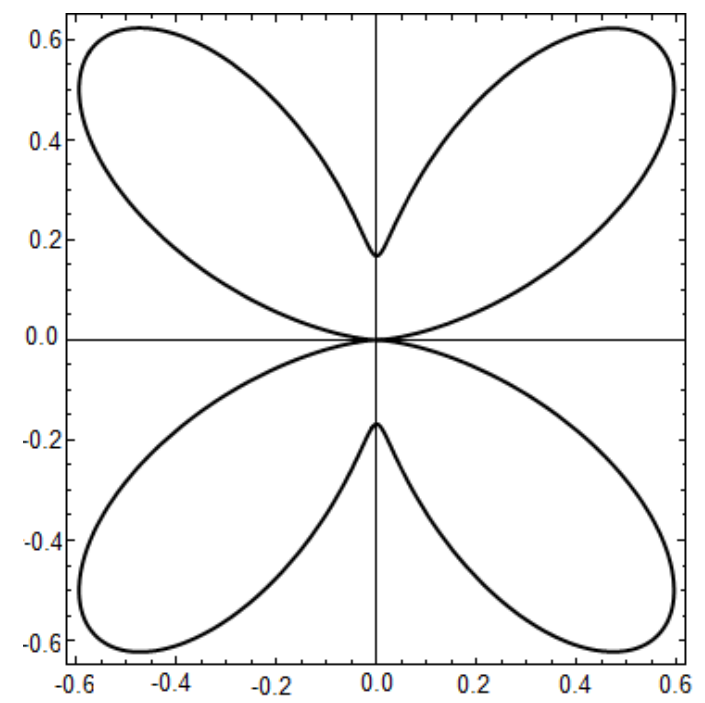

Fig. 5: Gráfica polar de la sección eficaz de dispersión normalizada $W_{437} / \Sigma_{437}$ para el segundo modo SH antisimétrico con el sexto modo Lamb antisimétrico dada por la ecuación (20) cuando $\theta \in[0,2 \pi]$.

Finalmente, en la Figura 6 se observa que cuando la energía entrante es un modo Lamb, los patrones de radiación de dispersión son isotrópicos. La isotropía y anisotropía en la dispersión, depende fundamentalmente de los valores que tomen los números de onda $\mathrm{k}_{\mathrm{i}}$, como también depende de la forma del modo entrante y dispersado, respectivamente.

Las gráficas polares de las secciones eficaces de dispersión obtenidas en este artículo son semejantes y en algunos casos idénticas a las obtenidas por Turner (Turner, 1998) para la dispersión de ondas sísmicas $\mathrm{P}$ y $\mathrm{S}$, en la corteza terrestre, por lo tanto, los resultados aquí obtenidos muestran la posibilidad de establecer analogías entre estos dos tipos de sistemas y usar el modelo de Trujillo y colaboradores para granulares por ejemplo para intentar la prevención de terremotos y deslizamientos de tierra. Además ya que algunos métodos de exploración de hidrocarburos en el subsuelo se basan en el análisis de ondas mecánicas previamente aplicadas y posteriormente detectas del subsuelo, después de que estas son múltiplemente dispersadas debido a las heterogeneidades del medio, el tipo de resultados aquí obtenidos podría entonces servir también para ayudar a obtener la configuración interna del subsuelo e inferir información relevante de su interior. Por otra parte, un problema de bastante relevancia en ingeniería, tiene que ver con la estabilidad de los silos y almacenes de granos, los cuales se rompen y colapsan debido a la forma aleatoria como se propagan los pesos de los granos a través del sistema, de tal forma que en ocasiones gran parte del peso total del sistema granular, puede concentrarse en algún punto de las paredes del silo ocasionando de esta forma el rompimiento y posterior colapso del mismo. Por lo tanto entender cómo se propaga la energía al interior del granular podría ayudar en el logro de la estabilidad de dichos sistemas. 


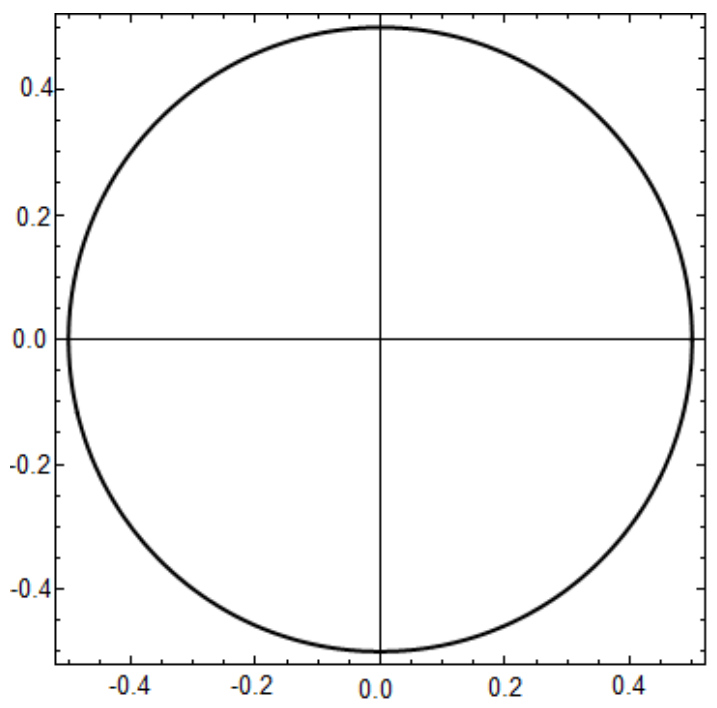

Fig. 6: Gráfica polar de las secciones eficaz de dispersión modal normalizadas $W_{2424}, W_{2411}, W_{246}, W_{2427}$. $W_{2515}, W_{254}, W_{2524}$ y $W_{2527}$, cuando $\theta \in[0,2 \pi]$, dadas por las ecuaciones [(21) - (28)].

El modelo de Trujillo y colaboradores predice que las ondas elásticas pueden transportarse a través de un paquete granular en forma difusiva y proporcionan la respectiva ecuación que permite el cálculo de la constante de difusión correspondiente. Para la obtención de esta constante de difusión, es necesario el cálculo previo de las distintas secciones eficaces de dispersión modal $\mathrm{W}_{\mathrm{ij}}$ calculadas en el presente trabajo, ya que estas representan los bloques fundamentales que constituyen el fenómeno de dispersión múltiple y transporte de una perturbación ondulatoria elástica a través del sistema granular. Esto es, de acuerdo con el modelo de Trujillo y colaboradores la ecuación para la constante de difusión está en función de todas las secciones eficaces $\mathrm{W}_{\mathrm{ij}}$. Será el propósito de futuros trabajos en esta línea el cálculo de la mencionada constante de difusión a partir de los resultados obtenidos en el presente trabajo para luego comparar los resultados con los experimentos de Jia, X. dados en las referencias (Jia et al., 2009; Jia, 2004), en los cuales ellos justamente encontraron como ya se mencionó en la introducción que el ultrasonido se propaga a través de un paquete granular de manera difusiva obteniendo un valor para la mencionada constante de difusión. El conocimiento de la constante de difusión, da información de la trayectoria libre media de dispersión, asociada a la múltiple dispersión de las ondas elástica a través del sistema, ese parámetro da cuenta de cómo es la configuración interna de los dispersores dentro del medio granular, lo cual, permite caracterizar dicho sistema.

Por último, cabe resaltar que todos los cálculos se hicieron considerando el ángulo de incidencia $\theta_{i}=0^{\circ}$, si se asumiera otro valor, las gráficas tendrían un comportamiento semejante, con la diferencia que aparecerá rotada de acuerdo al ángulo de incidencia que se asigne.

\section{CONCLUSIONES}

Después de haber realizado un análisis exhaustivo de los resultados obtenidos, se puede concluir que:

i) Se realizó el cálculo de dieciséis de mil seiscientos (16/1600), secciones eficaces de dispersión modal normalizadas y sus graficas polares correspondientes, logrando con esto mostrar cómo se comporta de manera dispersiva la energía elástica en un paquete granular, es decir, en qué direcciones preferenciales se dan las diferentes transiciones de energía.

ii) Con los resultados obtenidos en este trabajo, se contribuye a un mejor entendimiento de la mecánica estructural de un paquete granular, ya que al conocer cómo se comporta la energía elástica en estos sistemas, se puede obtener información necesaria y suficiente de su estructura interna. Con lo anterior, se pretende contribuir al desarrollo de nuevas tecnologías de métodos no destructivos para la caracterización de materiales.

iii) Con esta contribución investigativa, se logra brindar herramientas necesarias, que aporten al desarrollo científico de disciplinas como, la mecánica de suelos para la prevención de terremotos y deslizamientos de tierra, exploración de hidrocarburos, la estabilidad estructural de sistemas de almacenamientos de granos, entre otros. 
iv) Con base en los resultados obtenidos en este trabajo y siguiendo el modelo de Trujillo y colaboradores, se puede determinar en futuros trabajos de investigación en esta línea, una constante de difusión que permita dar información de la caracterización del sistema.

\section{REFERENCIAS}

Carter S., J. Bowman, N. Handy: Extensions and tests of "multimode": a code to obtain accurate vibration/rotation energies of many-mode molecules, pp 191-198 (1998)

Forterre, Y. y O. Pouliquen, Granular Flows, Glasses and Grains, Poincaré Seminar 2009, Progress in Mathematical Physics, Springer Basel AG, Vol. 61, pp 77 - 109, Paris, France (2011)

Go N., T. Noguti, and T. Nishikawa: Dynamics of a small globular protein in terms of low- frequency vibrational modes, Vol 80, pp 3696-3700 (1983)

Gudehus G., Y. Jiang y M. Liu: Seismic- and thermodynamics of granular solids. Granular Matter, 13, 319$340(2011)$

Harrison E. R. Normal Modes of Vibrations of the Universe, Reviews moderns Physics, Vol 39, No 4, pp 862882, (1967)

Hutchinson John S., William P. Reinhardt, and James T. Hynes: Nonlinear resonances and vibrational energy flow in model hydrocarbon chains, The Journal of Chemical Physics 79, 4247 (1983)

Jia, X.: Coda like multiple scattering of elastic waves in dense granular media. Phys. Rev. Lett.:93(15), 154303: 1 - 4 (2004)

Jia, X., J. Laurent, Y. Khidas y V. Langlois: Sound scattering in dense granular media, Chinese Science Bulletin: 54(23), 4327 - 4336, December, (2009)

Jiang, Y., Liu, M.: A brief review of "granular elasticity", why and how far is sand elastic?. Eur. Phys. J. E 22, 255-260 (2007)

LangloisV. y X. Jia:Ultrasonic monitoring of the elastic properties of PMMA bead packings and their rearrangement during pressure sintering. Powder Technology, 208, 509-514 (2011)

Lawton R.T. \& M.S. Child:Local mode vibrations of water, Molecular Physics: An International Journal at the Interface Between Chemistry and Physics, 37:6, 1799-1807(1979)

Li Robert, J. Melngailis: The Influence of Stored Energy at Step Discontinuities of the Behavior of SurfaceWave Gratings, Vol 22, No 3, pp 189-198 (1975)

Mills I.M. y A.G. Robiette: On the relationship of normal modes to local modes in molecular vibrations, Molecular Physics: An International Journal at the Interface Between Chemistry and Physics, 56:4, 743765(1985)

Moreau L., M. Caleap, A. Velichko, P. Wilcox: Scattering of guided waves by through-thickness cavities with irregular shapes, Wave Motion, 48(7), 586-602 (2011)

Planès T., Larose E.: A review of ultrasonic Coda Wave Interferometry in concrete. Cement and Concrete Research, 53, 248-255 (2013)

Shaw S., C. Pierre: Normal Modes of Vibration for Non- Linear ContinuousSystems, Journal of Sound and Vibration 169(3), pp 319-347 (1994)

Toda M. Vibration of a Chain with Nonlinear Interaction, Journal of the Physical Society of Japan, Vol. 22, No 2, pp 431- 436 (1967)

Trujillo, L., L. Di G. Sigalotti y J. Klapp:Granular Hydrodynamics, Fluid Dynamics in Physics, Engineering and Environmental Applications,Environmental Science and Engineering by Klapp, J. et al., pp $169-183$, Springer-Verlag Berlin, Heidelberg, Germany(2013)

Trujillo, L., F. Peniche y X. Jia: Multiple Scattering of Elastic Waves in Granular Media: Theory and Experiments. Waves in fluids and solids. (Ed. R. Pico Vila) In Tech, Chap. V, pp. 127 - 152, September, (2011)

Turner, J.: Scattering and Diffusion of Seismic Waves, Bulletin of the Seismological Society of America, Vol 88, No 1, pp 276-283 (1998)

Weaver, R. L.: Diffuse waves in finite plates. J. Sound and Vibration, 94(3), 319-335 (1984) 